\title{
Use of low-dose computed tomography to assess pulmonary tuberculosis among healthcare workers in a tuberculosis hospital
}

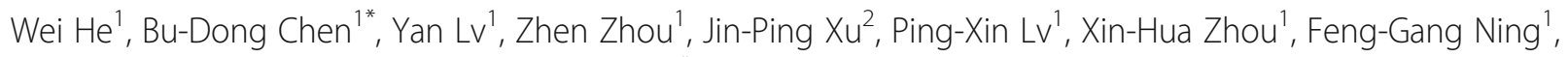
Cheng-Hai Li ${ }^{1}$, Dong-Po Wang ${ }^{1}$ and Jie Zheng ${ }^{3^{*}}$

\begin{abstract}
Background: According to the World Health Organization, China is one of 22 countries with serious tuberculosis (TB) infections and one of the 27 countries with serious multidrug-resistant TB strains. Despite the decline of tuberculosis in the overall population, healthcare workers (HCWs) are still at a high risk of infection. Compared with high-income countries, the TB prevalence among HCWs is higher in low- and middle-income countries. Low-dose computed tomography (LDCT) is becoming more popular due to its superior sensitivity and lower radiation dose. However, there have been no reports about active pulmonary tuberculosis (PTB) among HCWs as assessed with LDCT. The purposes of this study were to examine PTB statuses in HCWs in hospitals specializing in TB treatment and explore the significance of the application of LDCT to these workers.

Methods: This study retrospectively analysed the physical examination data of healthcare workers in the Beijing Chest Hospital from September 2012 to December 2015. Low-dose lung CT examinations were performed in all cases. The comparisons between active and inactive PTB according to the CT findings were made using the Pearson chi-square test or the Fisher's exact test. Comparisons between the incidences of active PTB in high-risk areas and non-high-risk areas were performed using the Pearson chi-square test. Analyses of active PTB were performed according to different ages, numbers of years on the job, and the risks of the working areas. Active PTB as diagnosed by the LDCT examinations alone was compared with the final comprehensive diagnoses, and the sensitivity and positive predictive value were calculated. (Continued on next page)
\end{abstract}

\footnotetext{
* Correspondence: budongchen@ccmu.edu.cn; zhengj@mir.wustl.edu ${ }^{1}$ Beijing Chest Hospital, Capital Medical University, No. 97 Beimachang, Tongzhou District, Beijing 101149, China ${ }^{3}$ Mallinckrodt Institute of Radiology, Washington University School of Medicine, 4525 Scott Ave, Room 3114, Saint Louis, MO 63110, USA Full list of author information is available at the end of the article
} International License (http://creativecommons.org/licenses/by/4.0/), which permits unrestricted use, distribution, and reproduction in any medium, provided you give appropriate credit to the original author(s) and the source, provide a link to the Creative Commons license, and indicate if changes were made. The Creative Commons Public Domain Dedication waiver (http://creativecommons.org/publicdomain/zero/1.0/) applies to the data made available in this article, unless otherwise stated. 
(Continued from previous page)

Results: A total of 1012 participants were included in this study. During the 4-year period of medical examinations, active PTB was found in 19 cases, and inactive PTB was found in 109 cases. The prevalence of active PTB in the participants was $1.24 \%, 0.67 \%, 0.81 \%$, and $0.53 \%$ for years 2012 to 2015 . The corresponding incidences of active PTB among the tuberculosis hospital participants were $0.86 \%, 0.41 \%, 0.54 \%$, and $0.26 \%$. Most HCWs with active TB (78.9\%, 15/19) worked in the high-risk areas of the hospital. There was a significant difference in the incidences of active PTB between the HCWs who worked in the high-risk and non-high-risk areas (odds ratio [OR], 14.415; 95\% confidence interval (C): 4.733 - 43.896). Comparisons of the CT signs between the active and inactive groups via chi-square tests revealed that the tree-in-bud, cavity, fibrous shadow, and calcification signs exhibited significant differences $(P=0.000,0.021,0.001$, and 0.024, respectively). Tree-in-bud and cavity opacities suggest active pulmonary tuberculosis, whereas fibrous shadow and calcification opacities are the main features of inactive pulmonary tuberculosis. Comparison with the final comprehensive diagnoses revealed that the sensitivity and positive predictive value of the diagnoses of active PTB based on LDCT alone were 100\% and 86.4\%, respectively.

Conclusions: Healthcare workers in tuberculosis hospitals are a high-risk group for active PTB. Yearly LDCT examinations of such high-risk groups are feasible and necessary.

Keywords: Tuberculosis, Pulmonary, Active tuberculosis, Healthcare workers, Low-dose computed tomography, Computed tomography

\section{Multilingual abstracts}

Please see Additional file 1 for translations of the abstract into the five official working languages of the United Nations.

\section{Background}

Active tuberculosis (TB) is an infectious disease caused by the bacteria Mycobacterium tuberculosis (M. tuberculosis) and can spread from person to person through the air. M. tuberculosis complex are transmitted by droplet infection in addition to rarely being spread via smear infections on the skin and mucous membranes, via contaminated dust particles or cuts and stab wounds involving contaminated cannulae or scalpels [1].

$\mathrm{TB}$ is a major global health problem. There were an estimated 9.6 million incident cases of TB in 2014 of which 5.4 million were adult men, 3.2 million were adult women, and 1.0 million were children [2]. Moreover, there were an estimated 1.5 million deaths from TB [2]. The number of TB cases relative to population size (i.e., the incidence rate) varies widely among countries. The TB burden is expressed in terms of the estimated annual incidence, mortality, and disease prevalence. These three outcome measures are reported per 100000 people. Estimates from the World Health Organization (WHO) are derived from population-based national surveys of the prevalence of $\mathrm{TB}$, time series of case notifications, and mortality data from vital registration systems with the standard coding of the causes of death. Most highTB burden countries that collectively account for $80 \%$ of TB cases have rates of approximately 150 - 300 cases per 100000 population per year [3]. According to the WHO, China is one of the 22 countries with serious TB infection and one of the 27 countries with serious multidrug-resistant TB strains [3]. In 2014, the number of TB infections in China was approximately 930000 , which accounted for nearly $10 \%$ of all global infections [2]. A total of $147941 \mathrm{~TB}$ cases were reported from 2009 to 2012 in Zhejiang Province alone [4].

Globally, TB prevalence in 2015 was $42 \%$ lower than that in 1990 [2]. However, despite the decline of TB in the overall population, healthcare workers (HCWs) remain at a high risk of infection [5]. A subsequent meta-analysis estimated that the average annual risk of developing TB disease was three times higher (95\% CI: 2.43 - 3.51) for HCWs (across all settings) compared to the general population [6]. The median annual incidence of TB among HCWs was 67, 91, and 1180 cases/100 000 persons in studies from countries with low, intermediate, and high TB incidence, respectively. The corresponding median TB incidences for the general populations were 33,82 , and 311 cases/100 000 persons [5]. Compared with high-income countries, the TB prevalence among HCWs from low- and middle-income countries are higher $[7,8]$. A systematic review of the TB incidence in low- and middle-income countries estimated that the annual risk of TB infection among HCWs ranges from $3.9 \%$ to $14.3 \%$ (with between $2.6 \%$ and $11.3 \%$ of cases attributable to occupational exposure) [7]. The early detection and treatment of TB in HCWs has important clinical significance.

Few studies have evaluated the risk of active pulmonary TB (PTB) among HCWs in China. It has been reported that tuberculin skin test (TST) reactions $\geq 5 \mathrm{~mm}$ occur in 69\% of the HCWs in Inner Mongolia, China [9]. A prospective cohort study that enrolled HCWs in a tertiary general hospital in Beijing, China revealed that 29/ $101 \mathrm{HCWs}$ (28.7\%) received positive diagnoses based on 
T-SPOT. TB and 53/101 (55.2\%) were positive according to TST (using a $\geq 10 \mathrm{~mm}$ cut-off) [10]. At present, active TB detection in high-risk groups primarily relies on X-ray. Low-dose CT (LDCT) is becoming more popular due to its superior sensitivity and lower radiation dose. However, there has been no reporting regarding active pulmonary tuberculosis among HCWs as detected by LDCT. The purpose of this study is to examine the active PTB statuses of HCWs in hospitals specializing in TB treatment. In this project, the physical examination results from HCWs working at the Beijing Chest Hospital over a period of 4 years $(2012-2015)$ were retrospectively analysed. These analyses included all LDCT scanning data.

\section{Methods}

\section{Participants}

We retrospectively analysed the health examination data of healthcare workers from the Beijing Chest Hospital affiliated with Capital Medical University from January 2012 to November 2015. The inclusion criterion were the following: 1) at least one LDCT examination; 2) age $>18$ years old; 3 ) If a $\mathrm{HCW}$ underwent 2 to $4 \mathrm{LDCT}$ examinations within the four years, that HCW was considered 1 participant; and 4) if a HCW was diagnosed as a TB patient in a particular year, he/she was rescanned in the next year, but he/she was counted as one new case only in the year of the initial diagnosis year. The exclusion criteria were as follows: 1) CT examination with the conventional dosage and 2) During pregnancy. This study was approved by the Medical Ethics Committee of the Beijing Chest Hospital affiliated with Capital Medical University. All participants provided written informed consent before participating in the study.

\section{Low-dose CT image acquisition}

All LDCT scans were performed using an Optima CT 680 Quantum or Lightspeed VCT (General Electric Company,
GE, America) scanners. The scanning scheme involved employed two types. Four hundred participants were scanned with a fixed tube voltage $(120 \mathrm{kV})$ and current (50 $\mathrm{mA})$, and 612 participants were scanned with automatic dose adjustment. For the latter type, the dose range was $10-80 \mathrm{~mA}$, and the average tube voltage was $120 \mathrm{kV}$. The scans were performed with spiral data acquisition and the following additional acquisition parameters: pitch, 1.375; and noise factor, 25 . For the LDCTs, all the images were reconstructed into axial images with $5 \mathrm{~mm}$ slice thickness at $5 \mathrm{~mm}$ intervals with a lung and mediastinal window algorithm and a $1.25 \mathrm{~mm}$ thin-section lung window using a lung reconstruction algorithm.

\section{Image analysis and classification of TB}

All CT scans and medical records were retrospectively reviewed by two chest radiologists with 16 and 20 years of experience. The two radiologists independently and blindly analysed the CT scans for the presence or absence and extent of the TB features. The resolution of differences in the observed findings was based on a consensus between the two radiologists. The airway abnormalities, lung abnormalities, pleura abnormalities, and lymph node enlargements were recorded.

According to the literature [11-13], the signs of PTB activity on CT include the following: tree-in-bud and centrilobular nodules, lobular or segmental or lobar/subsegmental consolidations, ground glass opacities, thick wall cavities, bronchial wall thickenings and masses $(>3 \mathrm{~cm})$. The signs of inactive PTB were fibrous and calcified lesions. Combined with the guidelines of the Health of the People's Republic of China Industry Standards for Tuberculosis [14], the PTB diagnostic criteria for the physical examinations are provided in Table 1 .

Table 1 Criteria for TB diagnosis in the physical examination

\begin{tabular}{|c|c|c|}
\hline Classification & Definition & Diagnosis \\
\hline Active PTB & $\begin{array}{l}\text { (1) smear-positive and culture-positive tuberculosis in sputum and bronchial lavage } \\
\text { fluid; pathological diagnosis for tuberculosis in the lung lesions } \\
\text { (2) although } 3 \text { sputum smear-negative tests, chest radiographic examination showing active } \\
\text { tuberculosis in the lesions } \\
\text { (3) tuberculosis suspicious symptoms such as cough, expectoration, hemoptysis; } \\
\text { (4) strong positive tuberculin test; } \\
\text { (5) anti-tuberculosis antibody examination positive; } \\
\text { (6) extrapulmonary histopathological examination confirming tuberculosis; } \\
\text { (7) anti-inflammatory therapy invalid and antituberculosis diagnostic treatment or follow-up } \\
\text { show valid. }\end{array}$ & (1) or $(2)+(3)-(7)$ in any of the terms \\
\hline Inactive PTB & $\begin{array}{l}\text { (1) residual lesions are stable for more than six months or gradual fibrosis and } \\
\text { calcification occurs in persons who were previously diagnosed with active } \\
\text { tuberculosis and cured by effective anti-tuberculosis treatment; } \\
\text { (2) lack of a history of active tuberculosis diagnosis, no symptoms } \\
\text { such as cough, sputum, or hemoptysis and sputum tuberculosis bacterium negative } \\
\text { but chest CT showed lesions that conforms to PTB that in common locations of } \\
\text { pulmonary tuberculosis, and the lesions did not change for more than } 6 \text { months } \\
\text { without anti-tuberculosis treatment. }\end{array}$ & $(1)$ or $(2)$ \\
\hline
\end{tabular}




\section{Classification of the work areas}

High-risk areas were defined as the working areas in the hospital in which diagnosed or undiagnosed patients with TB were likely to be cared for. These areas included TB wards, multidrug-resistant TB wards, outpatient departments, TB outpatient clinics, general wards, and radiography clinics. Intermediate-risk areas were defined as working areas in the hospital in which there was a probability of having contact with patients with TB and included laboratories. The low-risk areas were defined as the working areas in the hospital in which there was little or no probability of having contact with patients and included administrative offices, finance departments, and libraries.

\section{Statistical analysis}

The LDCT examination results were analysed using the Statistical Package for Social Sciences (SPSS) version 19.0. The comparisons between active and inactive PTB based on the CT findings were performed using the Pearson chi-square test or the Fisher's exact test. Comparisons between the incidences of active PTB in the high-risk areas and the non-high-risk areas were performed using the Pearson chi-square test. The analyses of active PTB were performed according to different ages $(20-25,26-$ $30,31-35,36-40,41-45,46-50,51-55,56-60$, $55-60$, and $>61$ years old), different numbers of years on the job $(0-5,6-10,11-15,16-20,>21)$, and different working risk areas (high risk, intermediate risk, and low risk areas). The active PTB diagnoses based on the LDCT examinations alone were compared with the final comprehensive diagnoses, and the sensitivity and positive predictive value were calculated. $P$ values less than 0.05 were considered statistically significant.

\section{Results}

\section{CT examination results}

The numbers of HCWs in the hospital in 2012, 2013, 2014, and 2015 were 970, 970, 995, and 982, respectively. The numbers of participants in 2012, 2013, 2014, and 2015 were $809,740,743$, and 752, respectively. A total of 1012 participants were included in this study. There were 340 males and 672 females, and the average age was $40.57 \pm 11.47$ years old $(18-82$ years old). Of the 1012 participants, 243 participants were under 30 years old, 240 were between 31 and 40 years old, 334 were between 41 and 50, 169 were between 51 and 60, and 26 cases were older than 60 years.

\section{PTB statuses based on the LDCT examinations}

From 2012 to 2015, 19 active PTB cases were found via LDCT examinations including 4 males and 15 females with a mean age of 39 years. Eighteen cases were diagnosed as secondary pulmonary tuberculosis including 2 sputum-positive bacteria and resistant tuberculosis cases and 1 case of mediastinal lymph node tuberculosis. The numbers of active PTB cases as assessed via annual CT examinations are provided in Table 2 . The prevalence rates of active PTB in the participants were $1.24 \%$ (10/ 809), $0.67 \%$ (5/740), $0.81 \%$ (6/743), and $0.53 \%$ (4/752). The incidences of active PTB in the participants were $0.86 \%$ (7/809), $0.41 \%$ (3/740), $0.54 \%$ (4/743), and $0.26 \%$ (2/752). Based on comparisons with the final comprehensive diagnoses, the sensitivity and positive predictive value of the diagnoses of active PTB based on LDCT alone were $100 \%$ and $86.4 \%$, respectively (Table 3). A total of 109 cases (10.9\%) had inactive PTB over the four years.

\section{Distributions of hospital participants with active pulmonary TB across the various departments}

Among the 19 active PTB cases, $3(3 / 19,15.8 \%)$ of the staff members did not work in clinical departments, and $2(2 / 19,10.5 \%)$ were paramedical staff who worked in laboratory conditions and as radiographers. However, 14 $(14 / 19,73.7 \%)$ were clinical staff (13 in the clinical departments that were in contact with $\mathrm{TB}$ patients and one in the oncology department, but this participant treated TB outpatients). The distributions active pulmonary TB in the hospital staff of the various departments are illustrated in Fig. 1, and the distributions of HCWs by age, years of employment, and working area are illustrated in Table 4. As indicated in Table 5, there was a significant difference in the incidence of active PTB between the HCWs who were working in the high-

Table 2 The numbers of active PTB participants diagnosed by LDCT examinations from $2012 \sim 2015$

\begin{tabular}{llllllll}
\hline Year & Total subjects & Ilness $^{c}$ & Incidence $^{\mathrm{e}}$ & Post-primary PTB $^{\text {(n) }}$ & Mediastinal LND TB $^{\text {a }}$ & Sputum positive $^{\text {Sputum negative }}$ \\
\hline 2012 & 809 & $10^{\text {d }}$ & 7 & 10 & 0 & 1 & 9 \\
2013 & 740 & 5 & 3 & 5 & 0 & 0 & 5 \\
2014 & 743 & 6 & 4 & 5 & 1 & 1 & 5 \\
2015 & 752 & 4 & 2 & 4 & 0 & $1^{\text {b }}$ & 3 \\
\hline
\end{tabular}

${ }^{a}$ Mediastinal LND TB: mediastinal lymph node TB

${ }^{b}$ One patient was sputum-positive with active PTB and MDR-TB in 2014 and remained sputum-positive in 2015

${ }^{\mathrm{c} C u r r e n t}$ illness

${ }^{d}$ Ten cases exhibited pulmonary TB in 2012, including 7 newly discovered active tuberculosis cases and 3 previously diagnosed active tuberculosis cases who were receiving treatment cases

eIncidence: the number of people with active PTB that was newly diagnosed based on LDCT 
Table 3 Comparison of the active PTB cases diagnosed based on LDCT examinations alone and based on the final comprehensive diagnosis

\begin{tabular}{llll}
\hline LDCT & \multicolumn{2}{l}{ Final Comprehensive diagnosis } & Total \\
\cline { 2 - 3 } & Yes & No & \\
\hline Yes & 19 & 3 & 22 \\
No & 0 & 990 & 990 \\
Total & 19 & 993 & 1012 \\
\hline
\end{tabular}

risk and non-high-risk areas $\left(\chi^{2}=33.901, P=0.000\right.$; odds ratio $[O R]=14.415,95 \% C I: 4.733-43.896)$.

\section{CT signs of pulmonary TB according to the LDCT examinations}

The main CT signs of active PTB discovered by LDCT from 2012 to 2015 are provided in Table 6. The comparison of the main CT signs discovered in the 19 active and 109 inactive PTB cases via LDCT is illustrated in Table 7. The comparisons between the two groups via chi-square tests revealed that the tree-in-bud $(P=0.000)$, cavity $(P=0.021)$, fibrous shadow $(P=0.001)$ and calcification $(P=0.024)$ signs exhibited significant differences. No significant differences were found for the other lesions.

A spiral CT in case 1 (Fig. 2a-d) illustrates an apicoposterior active, multidrug-resistant PTB in the left lung of a 42-year-old female patient. Panels A through D illustrate chest CT scan images collected from 2012 to
2015, respectively. Micronodules, ground glass opacity, and fibrous stripes shadow at the pulmonary window were observed in the left apicoposterior segment in 2012 (a, white arrow) and 2013 (b). Because there were no clinical symptoms, the TB was considered inactive. However, the micronodular shadows in the left upper apicoposterior segment grew with tree-in-bud opacities and micronodules shadow in 2014 (c, yellow arrow). The final diagnosis was active and multi-drug resistant TB. After a year of treatment, the micronodular shadows decreased in 2015 (d).

A spiral CT from case 2 (Fig. 3a-d) illustrates a case of active PTB in the right lower lung in a 40-year-old female. The chest CT scan images in panels A through D were collected in 2012, 2013, and 2015, respectively. The lung was clear in 2012 (a) and 2013 (b). There was no physical examination in 2014. However, tree-in-bud nodules and centrilobular nodules in the pulmonary window (c, white arrow) were observed in the superior segment of the right lower lung in 2015. After three months of treatment, the tree-in-bud nodules and the lobular center nodules decreased (d).

A spiral CT from case 3 (Fig. 4a-d) illustrates active PTB in the right upper lung of a 36-year-old male. The chest $\mathrm{CT}$ scans $(\mathrm{a}-\mathrm{d})$ illustrate the $\mathrm{CT}$ manifestations from 2012 to 2015. Two micronodules (a, white arrow) at the pulmonary window were observed in the right upper posterior segment in 2012. We recommended anti-inflammatory treatment and ruled out active TB,



Fig. 1 The population distributions of active pulmonary TB in the hospital staff across the various departments 
Table 4 The numbers of active PTB cases from 2012 2015 separated according age, length of employment, and working area

\begin{tabular}{lll}
\hline & $\begin{array}{l}\text { Number of } \\
\text { active TB }\end{array}$ & $\begin{array}{l}\text { Percentage of } \\
\text { active TB(\%) }\end{array}$ \\
\hline $\begin{array}{lll}\text { Age (years) } \\
20-25\end{array}$ & 2 & 10.5 \\
$26-30$ & 3 & 15.7 \\
$31-35$ & 1 & 5.3 \\
$36-40$ & 4 & 21.1 \\
$41-45$ & 4 & 21.1 \\
$46-50$ & 2 & 10.5 \\
$51-55$ & 2 & 10.5 \\
$56-60$ & 1 & 5.3 \\
$>61$ & 0 & 0 \\
Length of employment (years) & & 10.5 \\
$0-5$ & 2 & 26.3 \\
$6-10$ & 5 & 26.3 \\
$11-15$ & 5 & 10.5 \\
$16-20$ & 2 & 26.4 \\
$>21$ & 5 & 78.9 \\
Work areas & & 5.3 \\
high-risk areas & & 15.8 \\
intermediate-risk areas & 15 & \\
low-risk areas & 3 & \\
\hline
\end{tabular}

but the patient did not receive any anti-inflammatory treatment. A small thickened wall cavitation with many nearby micronodules (b, yellow arrow) was observed at the same location in 2013. The final diagnosis was active TB. The cavitation was closed, and the micronodules disappeared or shrank by 2014 after one year of treatment (c). One micronodule and fibrous shadow were observed in 2015 were seen the same as in 2014 (d).

\section{Discussion}

For the first time, this study investigated the prevalence and departmental distributions of HCWs with active PTB in a hospital specializing in treating TB patients based on LDCT examinations. The incidence and prevalence rate of active TB per year were $>2.8$ times and $>4.1$ times greater than the average incidence and prevalence of TB in China in 2014, respectively. In 2014, China's TB

Table $\mathbf{5}$ Comparison of the incidences of active PTB cases in high-risk and non-high-risk areas

\begin{tabular}{llll}
\hline Areas & Active PTB & & Total \\
& Yes & No & \\
\hline High-risk & 15 & 205 & 220 \\
Non-high-risk & 4 & 788 & 792 \\
Total & 19 & 993 & 1012 \\
\hline
\end{tabular}

Table 6 The features of active PTB on LDCT images from 2012 to 2015

\begin{tabular}{lllll}
\hline & 2012 & 2013 & 2014 & 2015 \\
\hline Tree-in-bud & 3 & 1 & 1 & 2 \\
Cavity & 1 & 1 & 0 & 0 \\
Patchy opacity & 7 & 2 & 1 & 0 \\
Calcification & 1 & 0 & 0 & 0 \\
Nodules & 9 & 2 & 1 & 1 \\
Ground glass opacity & 3 & 1 & 0 & 0 \\
Lymph node enlargement & 0 & 0 & 1 & 0 \\
Total & 10 & 3 & 4 & 2 \\
\hline
\end{tabular}

incidence and prevalence rate were 68/100 000 and 98/ 100 000, respectively [2]. These observations are not surprising, given the relatively large population of TB patients seen in our hospital. There are over 100000 TB-related outpatient services performed annually, and 2600 TB-related patients are hospitalized. Frequent exposure to mycobacterium TB leaves HCWs at a high risk of infection with TB [6, 15-18].

According to our analysis (Fig. 1), most HCWs with active PTB $(78.9 \%, 15 / 19)$ worked in the high-risk areas. These HCWs were in frequent and close contact with TB patients and included 7 nurses and 6 physicians working in the outpatient division, TB ward and intensive care unit in addition to one CT room technician and one physician working in the oncology department who performed outpatient work with TB patients. However, only one $\mathrm{HCW}$ with active TB worked in an intermediate-risk area (laboratory), and three HCWs worked in low-risk areas. Tudor et al. [19] conducted a case-control study of HCWs who were diagnosed with TB in 3 district hospitals with specialized multidrug-resistant $\mathrm{TB}$ wards in KwaZulu-Natal, South Africa and found that cases occurred more frequently among the clinical staff $(46 \%)$ and the support staff (35\%). The study population

Table 7 Comparison of the features of active PTB and inactive PTB on LDCT images

\begin{tabular}{|c|c|c|}
\hline CT sign & Active PTB & Inactive PTB \\
\hline Tree-in-bud $^{a}$ & 7 & 0 \\
\hline Cavity $^{a}$ & 2 & 0 \\
\hline Patchy opacity & 10 & 34 \\
\hline Calcification $^{a}$ & 1 & 33 \\
\hline Nodules & 13 & 75 \\
\hline Ground glass opacity & 4 & 1 \\
\hline Lymph node enlargement & 1 & 1 \\
\hline Fibrous shadow ${ }^{a}$ & 8 & 85 \\
\hline Total & 19 & 109 \\
\hline
\end{tabular}

${ }^{\mathrm{a}}$ Significant difference between the active and inactive TB groups 


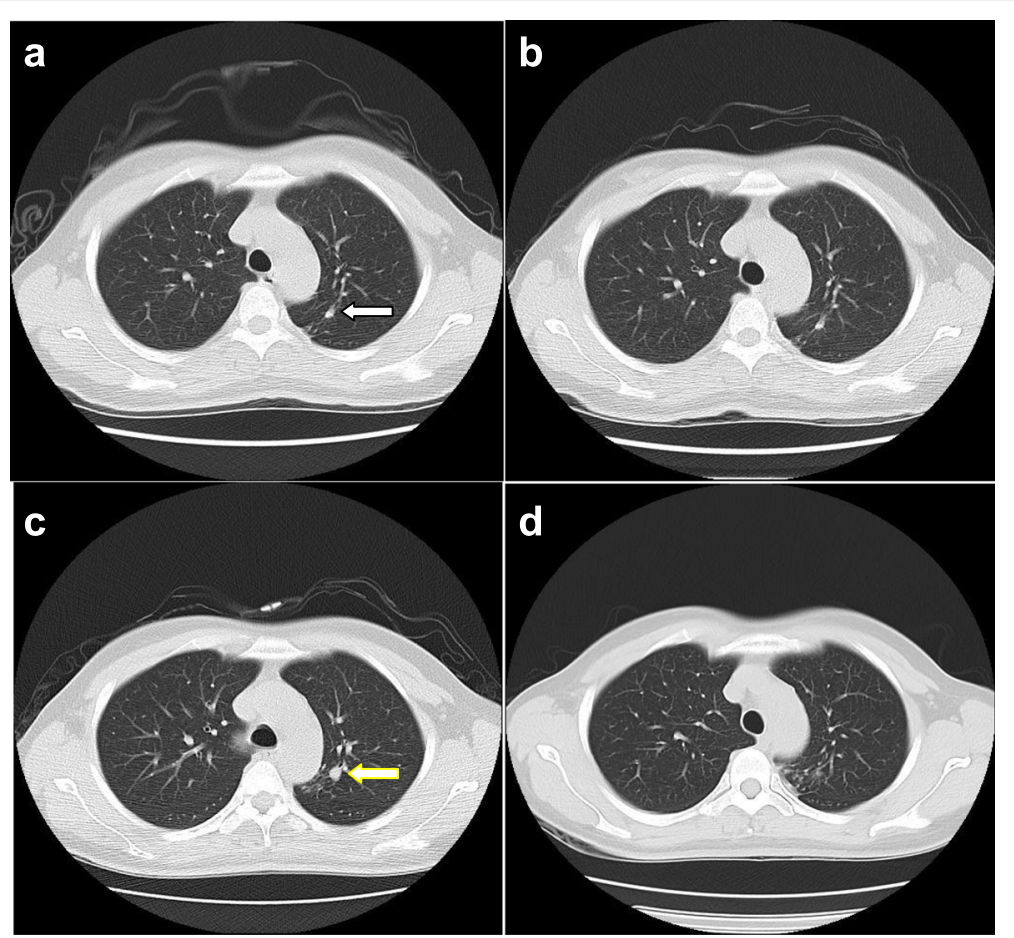

Fig. 2 a-d LDCT images of an active PTB case (Case 1) from 2012 to 2015

distribution was not exactly the same as the population distribution in the present study. The reasons could be that the personnel statistics are not exactly the same (the hospital staff in our study did not include orderlies and cleaners). From Table 5 , it is readily apparent that the incidence of active PTB in the high-risk areas was more than 13 times greater than that in the non-high-risk areas. In general, there is a strong need for the improvements to prevent and control infection among HCWs working in the high-risk areas of the hospital.

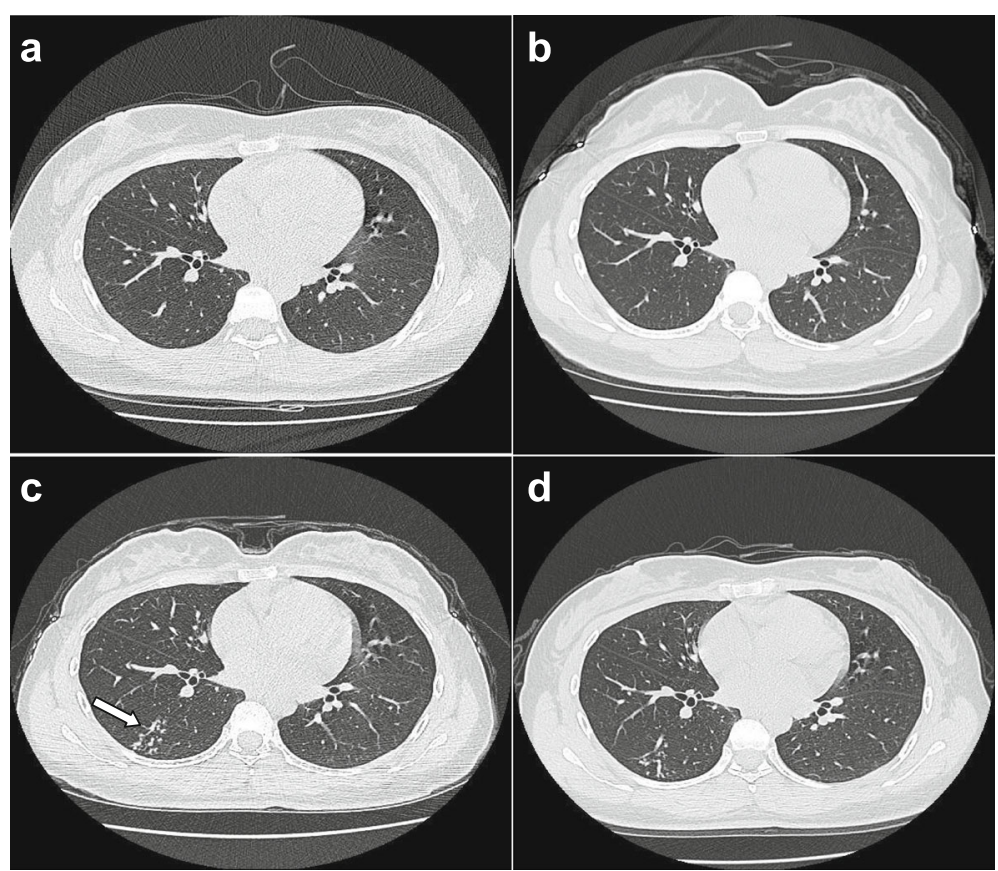

Fig. 3 a-d LDCT images of an active PTB case (Case 2) from 2012 to 2015 

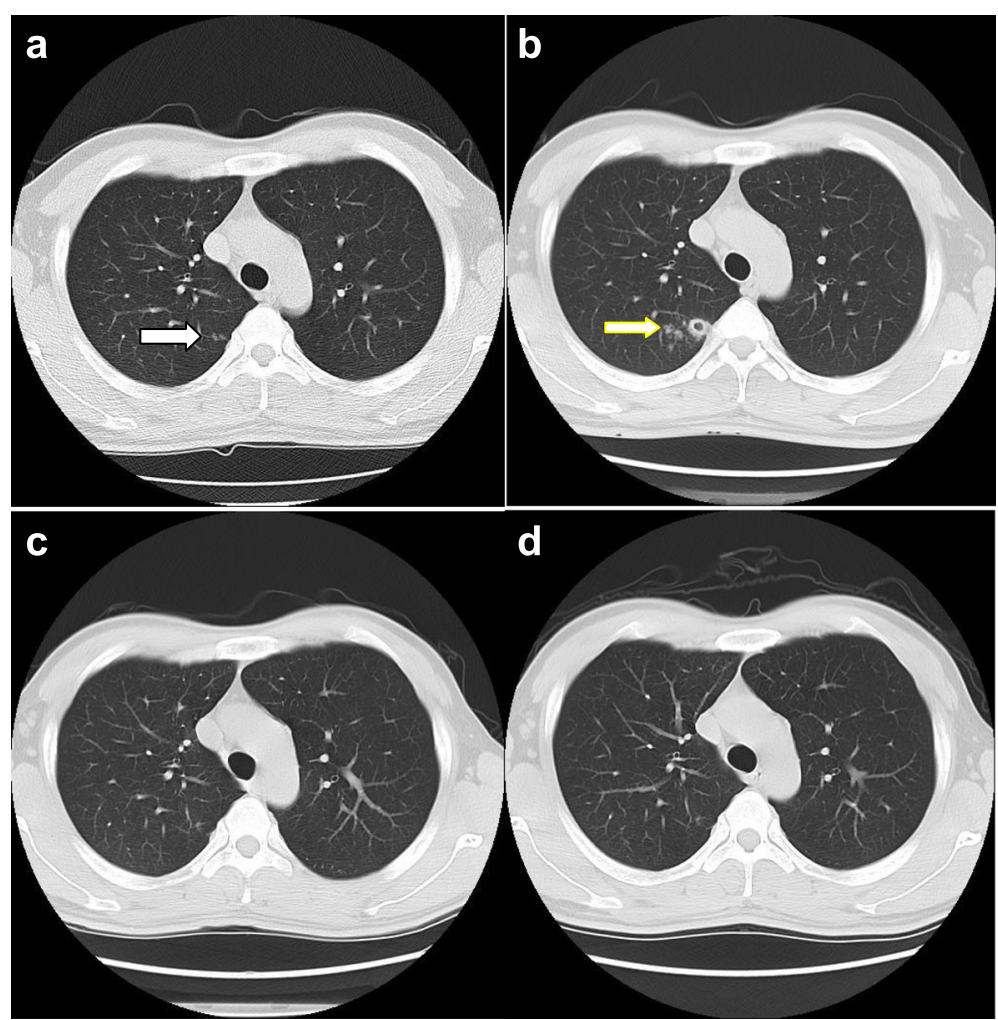

Fig. 4 a-d LDCT images of an active PTB case (Case 3) from 2012 to 2015

The average age of the discovered active PTB groups was 39 years old, and the peak occurred at $36-45$ years old (42.2\%). Moreover, the majority of these participants were women (15/19). The latter finding can be explained by the fact that the ratio of female staff to male staff was 2:1. Furthermore, middle-aged female HCWs have both household and work burdens in this society, which leaves them particularly vulnerable to TB infection. As noted in Table 4, the distribution of the percentages of HCWs who were affected with active TB was a relatively uniform across the different number of years of employments. These data suggest that the length of employment may not be a risk factor for TB infection, i.e., every HCW was susceptible to infection by active TB. Of the 19 active TB cases in our study, 2 (10.5\%) had multidrug-resistant $\mathrm{TB}$, which suggests a relatively high incidence among HCWs in TB hospitals. In 2014, the incidence of drug-resistant TB cases was estimated to be $5-7 \%$ in China [2]. Delays in the diagnoses, less effective treatments for drug-resistant $\mathrm{TB}$, and longer periods of contact with drug-resistant TB patients may have increased the potential for the transmission of drug-resistant strains to HCWs. Accordingly, HCWs are up to six times more likely to be hospitalized for drugresistant TB than the population they care for $[20,21]$.
In current practice, the diagnosis of active TB primarily relies on bacteriological inspection and $\mathrm{X}$-ray detection. Among pulmonary TB cases, $58 \%$ were bacteriologically confirmed (as opposed to clinically diagnosed) in 2014. This data was unchanged from 2013 [2]. Chest radiography (CXR) has been used for over a century to diagnose pulmonary TB. However, CXR is limited by modest specificity and high interobserver variability [22]. CXR presents a low yield in the detection of active TB [23].

Computed tomography (CT) is a corroborative imaging modality for the study of TB [24]. CT helps to distinguish between active and inactive disease and is more sensitive than CXR in the detection of both localized and disseminated disease and mediastinal lymphadenopathy [12, 21, 25]. Chest CT can effectively detect $80 \%$ of patients with active PTB and $89 \%$ of those with inactive PTB [26]. Compared to conventional-dose CT, the use of LDCT for active tuberculosis detection can obviously reduce the radiation exposure and damage to the body. However, the diagnosis of TB cannot be established by radiography alone. Results from 425 individuals from 6 different European centres revealed that the sensitivities of the novel tests TST, QuantiFERON-TB GOLD, In-TubeQuanti (QFT-GIT) and T-SPOT.TB were $73.1 \%, 85.3 \%, 78.1 \%$, and $85.2 \%$, respectively, and 
the specificities were $70.6 \%, 48.0 \%, 61.9 \%$ and $44.3 \%$, respectively [27]. In a contact investigation of a TB outbreak in a high school [28], TST and CXR were performed on all 1044 employees and students. QFT-G was performed on the TST-positive subjects, and CT was performed on the QFT-G-positive subjects and students with TST values of $\geq 20 \mathrm{~mm}$. The results revealed TST positivity in 388 subjects (37.2\%), while the QFT-G tests were positive in $7.6 \%$ of the subjects $(30 / 394)$. CXR exhibited abnormal findings for TB in $10(1.0 \%)$ subjects, all of whom were TST-positive, and six of whom were QFT-G-positive. Active PTB was noted in 17 (32.7\%) of 52 subjects by CT. Collectively, among $21(1.1 \%)$ TB patients, all were TST-positive, 12 (57.1\%) were QFT-Gpositive, and active TB was diagnosed by $\mathrm{CT}$ and not by CXR in 11 subjects. Our hospital is a specialized TB hospital. The working staff has close contact with the TB patients. Therefore, it is inadequate to use TST to screen for active TB.

From the LDCT results from the patients with active PTB, tree-in-bud opacities, and micronodules, the ground glass density shadows and lymph node enlargements were found to be small lesions that were of low density and/or were hidden within the mediastinum. Such lesions are easily missed by CXR but can be delineated by LDCT. The thick wall cavity and tree-in-bud opacities are the image features of active PTB, whereas the fibrous lesions and calcification are features of inactive TB. The three cases also demonstrated the importance of annual physical examinations and the significance of dynamic changes on LDCT for the differentiation of active and inactive TB.

Lew et al. demonstrated that no diagnostic test has $100 \%$ sensitivity for TB diagnosis and suggested a combined diagnostic approach that includes TST, CXR, IGRA, and CT [29].

Kowada $\mathrm{A}$ et al. found that a strategy involving QFT followed by HRCT yielded the greatest benefit at the lowest cost (\$US 6308.65; 27.56045 quality-adjusted lifeyears [QALYs]) [year 2012 values]. Cost-effectiveness was found to be sensitive to the BCG vaccination rate. HRCT chest imaging in the place of CXR is recommended as a cost-effective addition to the evaluation and management of TB contacts in public health policy [30]. In our practice, if the LDCT images revealed abnormalities in the HCWs working in high-risk areas, then active PTB would be suspected. Some of these HCWs exhibited no apparent symptoms, and some had only minor coughs in the present study. Using the final comprehensive diagnosis as the reference, the sensitivity of LDCT alone was $100 \%$, which clearly demonstrates the importance of LDCT in the annual check-up for TB among high-risk health professionals.

There are a few limitations to this retrospective study. Not all HCWs participated in the physical examinations annually. Some of the young HCWs with relatively few years of employment and those working in low-risk areas may not have participated in the annual physical examinations. This fact may have influenced the determination of the distributions of active PTB according to age and working area. In our physical examination, LDCT scans, rather than HRCT scans, were performed to evaluate micronodules and tree-in-bud opacities, which may have resulted in an underestimation of the presence of tiny cavitations or micronodules. Nonetheless, the resolution of LDCT has steadily improved, and this improvement will facilitate the evaluation of subtle abnormalities. Moreover, although all the staff did not participate in the LDCT examinations, the annual participation rates reached $75-84 \%$ among all hospital staff in those years year, and these values are sufficient to represent the active tuberculosis prevalence and incidence among the hospital HCWs. Additionally, the use of even lower-dose LDCT to detect TB remains an interesting research topic, and the comparison of the performance of QFT with LDCT images is an alternative in the future.

\section{Conclusions}

Due to their close contact with TB patients, the HCWs in a hospital specializing in the treatment of TB were at a high risk for active PTB. Annual LDCT examinations were important for discovering active PTB and distinguishing active from inactive TB disease. The government and hospital should adopt better prevention measures to protect HCWs.

\section{Additional file}

Additional file 1: Multilingual abstracts in the five official working languages of the United Nations. (PDF $765 \mathrm{~kb}$ )

\section{Abbreviations}

CXR: Chest radiography; HCWs: Healthcare workers; IGRA: Interferon-gamma release assay; LDCT: Low-dose computer tomography; PTB: Pulmonary tuberculosis; QFT: QuantiFERON-TB; TB: Tuberculosis; TST: Tuberculin skin test; WHO: World Health Organization

\author{
Acknowledgements \\ The authors are grateful to David Muccigrosso at Washington University \\ School of Medicine (USA) for editing and proofreading the manuscript. \\ Funding \\ Not applicable. \\ Availability of data and materials \\ Not applicable. \\ Authors' contributions \\ $\mathrm{WH}, \mathrm{BCH}$ and $\mathrm{JZ}$ participated in the design of the study and drafted the \\ manuscript. YL, ZZ, JPX, PXL, XYZ, FGN, CHL and DPW participated in the \\ case collection. $\mathrm{WH}, \mathrm{BDC}$ and $\mathrm{JZ}$ participated in the writing and revision of \\ the manuscript. All authors have read and approved the final manuscript.
}




\section{Competing interests}

The authors declare that they have no competing interests.

\section{Consent for publication}

I agree to publish the paper.

\section{Ethics approval and consent to participate}

The research was performed on data that was archived in our institute, and ethics approval and consent was given by the medical ethics committee of Beijing Chest Hospital, Capital Medical University under reference number (2016-02-02).

\section{Author details}

'Beijing Chest Hospital, Capital Medical University, No. 97 Beimachang, Tongzhou District, Beijing 101149, China. ${ }^{2}$ The school hospital, Beijing Science and Technology University, Beijing 100083, China. ${ }^{3}$ Mallinckrodt Institute of Radiology, Washington University School of Medicine, 4525 Scott Ave, Room 3114, Saint Louis, MO 63110, USA.

Received: 14 June 2016 Accepted: 5 March 2017

Published online: 24 March 2017

\section{References}

1. Diel R, Seidler A, Nienhaus A, Rusch-Gerdes S, Niemann S. Occupational risk of tuberculosis transmission in a low incidence area. Respir Res. 2005;6:35-45.

2. World Health Organization (WHO). Global tuberculosis report 2015. Geneva: WHO; 2015.

3. World Health Organization (WHO). Global tuberculosis report 2013. Geneva: WHO; 2013

4. Ge E, Zhang X, Wang X, Wei X. Spatial and temporal analysis of tuberculosis in Zhejiang Province, China, 2009-2012. Infect Dis Poverty. 2016;5(1):11.

5. Nienhaus A, Schablon A, Preisser AM, Ringshausen FC, Diel R. Tuberculosis in healthcare workers - a narrative review from a German perspective. J Occup Med Toxicol. 2014;9(1):9.

6. Baussano I, Nunn P, Williams B, Pivetta E, Bugiani M, Scano F. Tuberculosis among health care workers. Emerg Infect Dis. 2011:17(3):488-94.

7. Joshi R, Reingold AL, Menzies D, Pai M. Tuberculosis among health-care workers in low- and middle-income countries: a systematic review. PLoS Med. 2006;3(12), e494.

8. Seidler A, Nienhaus A, Diel R. Review of epidemiological studies on the occupational risk of tuberculosis in low-incidence areas. Respiration. 2005;72(4):431-46.

9. He GX, Wang LX, Chai SJ, Klena JD, Cheng SM, Ren YL, Ren LP, Gao F, Li YY, He GM, Li JB, Wang Y, Rao C, Varma JK. Risk factors associated with tuberculosis infection among health care workers in Inner Mongolia, China. Int J Tuberc Lung Dis. 2012;16(11):1485-91.

10. Zhang LF, Liu XQ, Zhang Y, et al. A prospective Evaluating longitudinal study to T-cell-based assay for latent tuberculosis infection in health-care workers in a general hospital in Beijing. Chin Med J(Engl). 2013;126(11):2039-44.

11. Poey C, Verhaegen F, Giron J, Lavayssiere J, Fajadet P, Duparc B. High resolution chest $C T$ in tuberculosis: evaluation patterns and signs of activity. Comput Assist Tomogr. 1997;21(4):601-7.

12. Im JG, Itoh H, Shim YS, Lee JH, Ahn J, Han MC, Noma S. Pulmonary tuberculosis: $C T$ findings-early active disease and sequential change with antituberculous therapy. Radiology. 1993;186(6):653-60.

13. Seon HJ, Kim Yl, Lim SC, Kim YH, Kwon YS. Clinical significance of residual lesions in chest computed tomography after anti-tuberculosis treatment. Int J Tuberc Lung Dis. 2014;18(3):341-6.

14. People's medical press:WS288-2008 - TB diagnosis standard - health industry standard of the People's Republic of China; 2008.

15. Kilinc O, Ucan ES, Cakan MD, Ellidokuz MD, Ozol MD, Sayiner A, Ozsoz MD. Risk of tuberculosis among healthcare workers: Can tuberculosis be considered as an occupational disease? Respir Med. 2002;96(7):506-10.

16. Baussano I, Bugiani M, Carosso A, Mariano D, Pia Barocelli A, Tagna M, Cascio V, Piccioni P, Arossa W. Risk of tuberculin conversation among healthcare workers and the adoption of preventive measures. Occup Environ Med. 2007:64:61-6.

17. Menzies D, Joshi R, Pai M. Risk of tuberculosis infection and disease associated with work in health care settings. Int J Tuberc Lung Dis. 2007; 11(6):593-605.
18. Hsi C, Chia-Jen S, Lee Y-J, Kuo SC, Hsu YT, Ou SM, Shih YN, Tarng DC, Li SY, Chen YT, Chen RC. Risk of tuberculosis among healthcare workers in an intermediate-burden country: a nationwide population study. J Infect. 2014;69(6):525-32.

19. Tudor C, Van der Walt ML, Margot B, Dorman SE, Pan WK, Yenokyan G, Farley JE. Occupational Risk Factors for Tuberculosis Among Healthcare Workers in KwaZulu-Natal, South Africa. Clin Infect Dis. 2016;62 Suppl 3:255-61.

20. Peng SS, Chan PC, Chang YC, Shih TT. Computed tomography of children with pulmonary Mycobacterium tuberculosis infection. J Formos Med Assoc. 2011;110(12):744-9.

21. O'Donnell MR, Jarand J, Loveday M, Padayatchi N, Zelnick J, Werner L, Naidoo K, Master I, Osburn G, Kvasnovsky C, Shean K, Pai M, Van der Walt M, Horsburgh CR, Dheda K. High incidence of hospital admissions with multidrug-resistant and extensively drug-resistant tuberculosis among South African health care workers. Ann Intern Med. 2010;153(8):516-22.

22. Sakurada S, Hang NT, Ishizuka N, Toyota E, Hung le D, Chuc PT, Lien LT, Thuong PH, Bich PT, Keicho N, Kobayashi N. Inter-rater agreement in the assessment of abnormal chest $\mathrm{X}$-ray findings for tuberculosis between two Asian countries. BMC Infect Dis. 2012;12:31.

23. Eisenberg RL, Pollock NR. Low yield of chest radiography in a large tuberculosis screening program. Radiology. 2010;256(3):998-1004.

24. Lee JY, Lee KS, Jung KJ, Han J, Kwon OJ, Kim J, Kim TS. Pulmonary tuberculosis: $C T$ and pathologic correlation. J Comput Assist Tomogr. 2000;24(5):691-8.

25. Jeong YJ, Lee KS. Pulmonary tuberculosis: up-to-date imaging and management. AJR. 2008;191(3):834-44.

26. Malaviya AN, Kapoor S, Garg S, Rawat R, Shankar S, Nagpal S, et al. Preventing tuberculosis flare in patients with inflammatory rheumatic diseases receiving tumor necrosis factor-alpha inhibitors in India. An audit report. J Rheumatol. 2009;36:1414-20.

27. Akira F, Tatsuya F, Satoshi M, et al. Tuberculosis Contact Investigation Using Interferon-Gamma Release Assay with Chest X-Ray and Computed Tomography. PLoS One. 2014;9(1), e85612.

28. Lew WJ, Jung YJ, Song JW, Jang YM, Kim HJ, Oh YM, et al. Combined use of QuantiFERON-TB Gold assay and chest computed tomography in a tuberculosis outbreak. Int J Tuberc Lung Dis. 2009;13:633-9.

29. Maeda T, Banno S, Maeda S, Naniwa T, Hayami Y, Watanabe M, Sato S, Ueda R. Comparison of QuantiFERON-TB Gold and the tuberculin skin test for detecting previous tuberculosis infection evaluated by chest $C T$ findings in Japanese rheumatoid arthritis patients. J Infect Chemother. 2011;17(6):842-8.

30. Kowada A. Cost effectiveness of high resolution computed tomography with interferon-gamma release assay for tuberculosis contact investigation. Eur J Radiol. 2013;82(8):1353-8

\section{Submit your next manuscript to BioMed Central and we will help you at every step:}

- We accept pre-submission inquiries

- Our selector tool helps you to find the most relevant journal

- We provide round the clock customer support

- Convenient online submission

- Thorough peer review

- Inclusion in PubMed and all major indexing services

- Maximum visibility for your research

Submit your manuscript at www.biomedcentral.com/submit
Biomed Central 